\title{
FRACTURE PROPERTIES OF AGGLOMERATED NANOPARTICLE REINFORCED POLYMERS: A COARSE-GRAINED MODEL
}

\author{
A. Mousavi ${ }^{1 *}$, B. Arash $^{1}$ and R. Rolfes ${ }^{1}$ \\ ${ }^{1}$ Institute of Structural Analysis, Leibniz University, Appelstraße 9A, 30167 Hannover, Germany. \\ *a.mousavi@isd.uni-hannover.de
}

Adding boehmite nanoparticles inside epoxy materials shows outstanding potential in improving the fracture properties. Coarse-grained models for pure epoxy and agglomerated boehmite nanoparticle/epoxy nanocomposites with initial single-edged cracks are developed. An optimization assisted modified iterative Boltzmann inversion method is proposed to calibrate coarse-grained force fields. Furthermore, the coarse-grained force field of nanoparticles is obtained using the strain energy conservation between coarse-grained models and all-atom systems [1]. In the proposed model, the energy release rate is obtained from the load-displacement curve of specimens [2]. Due to the size of the agglomerated boehmite nanoparticles, the molecular dynamic approach is not able to capture the fracture properties of agglomerated boehmite nanoparticle/epoxy nanocomposite; therefore, the existence of a coarse-grained model is crucial. The applicability of the coarse-grained model to estimate the fracture properties of the polymer reinforced nanocomposites is evaluated using experimental data. It is shown that the fracture properties of the nanocomposites depend on the weight fraction and distribution of nanoparticles. The dependence of the critical energy release rate on the initial crack length is also studied.

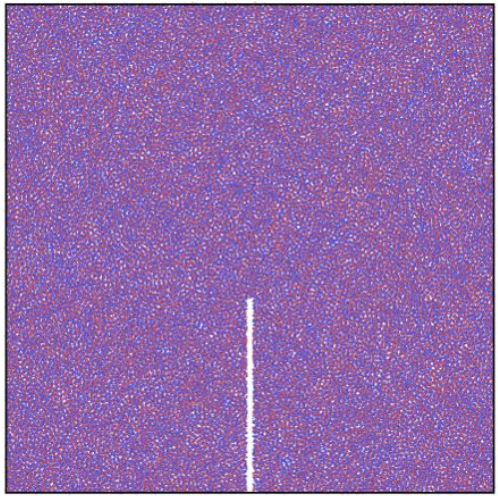

(a)

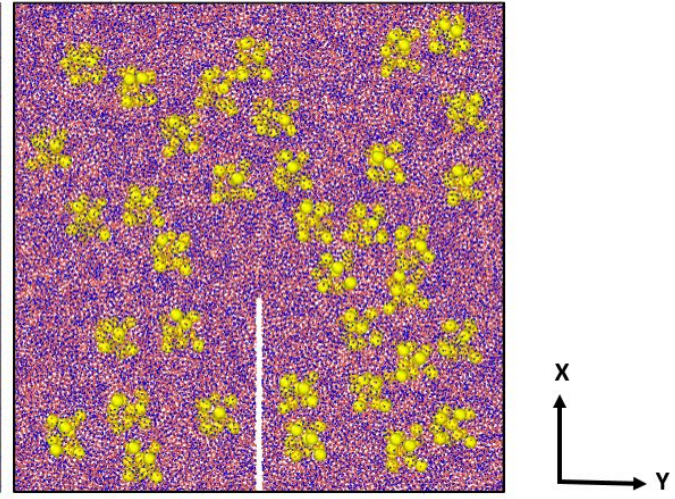

(b)

Figure 1: The top view of an RVE of (a) the pure epoxy and (b) an agglomerated Boehmite (15 wt\%)/epoxy nanocomposite with a size of $80 \times 80 \times 8 \mathrm{~nm}^{3}$ and 32-nm long initial notch in the middle in the y-direction. The CG model of the composite system contains 158560 beads that is equivalent to all-atom system with 5005992 atoms.

\section{References}

[1] B. Arash, H.S. Park and T. Rabczuk (2015) Mechanical properties of carbon nanotube reinforced polymer nanocomposites: A coarse-grained model. Composites Part B: Engineering, 80, pp.92-100.

[2] B. Arash, H.S. Park and T. Rabczuk (2016) Coarse-grained model of the J-integral of carbon nanotube reinforced polymer composites. Carbon, 96, pp.1084-1092. 